\title{
Segmental coupling effects during correction of three-dimensional lumbar deformity using lateral lumbar interbody fusion
}

\author{
Hiroto Yamaguchi $^{1,2} \cdot$ Hidetoshi Nojiri $^{1}\left[\right.$ C Kei Miyagawa ${ }^{1} \cdot$ Nozomu Inoue $^{3} \cdot$ Kazuo Kaneko $^{1}$
}

Received: 20 April 2019 / Revised: 5 October 2019 / Accepted: 18 January 2020 / Published online: 29 January 2020

(c) The Author(s) 2020

\begin{abstract}
Purpose Lateral lumbar interbody fusion (LLIF) has been performed to correct spinal deformity associated with lumbar degenerative disease. Although its usefulness has been studied, there are no reports of quantitative evaluation in three dimensions. Our purpose is to quantitate 3D deformity of the patients with lumbar degenerative disease and correction of the deformity by LLIF using patient-specific 3D CT models.

Methods We measured the disc height and 3D alignment of the lumbar spine in 28 patients with degenerative disease undergoing LLIF using patient-specific 3D CT models created preoperatively and 3 months after surgery. The 3D alignment was calculated as wedge, lordosis and axial rotation angles at each motion segment. The disc height and the rotational angles were compared between before and after LLIF.

Results A strong positive correlation was found between the wedge angle and the axial rotation angles $(r=0.718, P<0.001)$ in the patients with lumbar degenerative disease preoperatively. The wedge and axial rotation angles decreased after surgery $(P<0.001$ and $P<0.001$, respectively). A positive correlation was found between the corrected wedge angle and the corrected axial rotation angle $(r=0.46, P<0.001)$.

Conclusion The present study demonstrated positive correlations between the wedge deformity and the axial rotational deformity in the patients with lumbar degenerative disease. The axial rotational deformity was simultaneously corrected with LLIF only by leveling the intervertebral wedge deformity via cage insertion without additional correction procedure.
\end{abstract}

\section{Graphic abstract}

These slides can be retrieved under Electronic Supplementary Material.
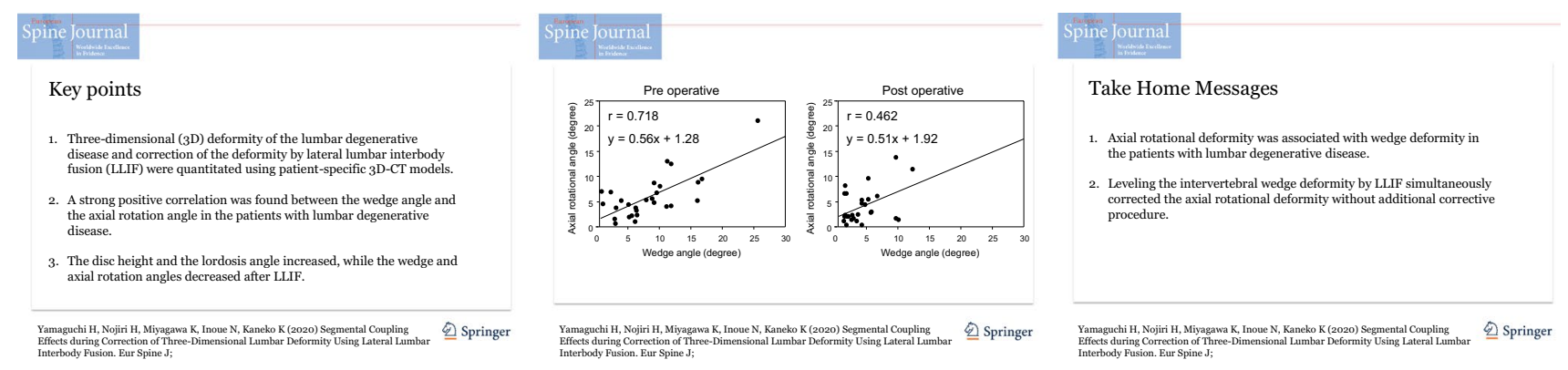

Keywords Degenerative lumbar scoliosis · Lumbar intervertebral deformity $\cdot$ Lateral lumbar interbody fusion (LLIF) $\cdot$ 3D alignment $\cdot$ In vivo 3D CT analysis

Electronic supplementary material The online version of this article (https://doi.org/10.1007/s00586-020-06310-0) contains supplementary material, which is available to authorized users.

Hidetoshi Nojiri

hnojiri@juntendo.ac.jp

Extended author information available on the last page of the article

\section{Introduction}

Degenerative lumbar scoliosis (DLS) is one of the most difficult clinical conditions to understand in the lumbar spinal disorders because its pathology and mechanism have not 
been fully elucidated. Deformity in DLS is primarily based on wedge disc deformity in a coronal plane; however, it has been considered to accompany axial rotation. Coexistence of the wedge and axial rotational deformity has been reported as one of the relevant factors of progression of the spinal deformity [1]. Currently, the various corrective instrumentation procedures have been performed even for highly degenerative scoliosis. However, indication and clinical results of corrective surgery for such three-dimensional (3D) deformity have been controversial [2].

Although importance of axial rotational deformity in DLS has been recognized, limited information on the segmental axial rotational angles in the DLS patients is available in the literature. The axial rotational deformity has been typically determined by means of evaluating positions of pedicles projected on plain radiograms [3]. Because of 3D nature of the spinal deformity in DLS, it is difficult to accurately measure rotational angles about three anatomical axes using two-dimensional (2D) image data.

In recent years, lateral lumbar interbody fusion (LLIF) has been applied for restoration of the disc height, correction of deformity and stabilization for DLS and its usefulness has been reported [4-6]. The LLIF procedure is a minimally invasive procedure in preserving anterior and posterior longitudinal ligaments intact. In the LLIF procedure, restoration of disc height causes tensioning of the anterior and posterior longitudinal ligaments, which is called "ligamentotaxis" and has been considered to provide posterior neural decompression, correction of spinal alignment and stabilization of the motion segment. Although some studies have addressed disc height restoration and deformity correction by LLIF, correlation between the disc height and the amount of deformity correction has not been analysed in a quantitative manner $[4,5]$.

The aim of the present study is twofold: first, to quantitate segmental rotational deformities in 3D and to clarify correlations between the rotational angles about three anatomical

Table 1 Demographic data of this study group

\begin{tabular}{ll}
\hline & Average $(n=28)$ \\
\hline Age & $69.5 \pm 9.2$ \\
BMI & $23.9 \pm 3.2$ \\
\hline & Number \\
\hline Sex & \\
Male & 11 \\
Female & 17 \\
Disease & \\
Spondylosis & 4 \\
Scoliosis & 16 \\
Spondylolisthesis & 8 \\
\hline
\end{tabular}

axes in patients with lumbar degenerative disease and second, to evaluate the effect of LLIF procedure on correction of 3D deformity using patient-specific 3D CT models.

\section{Materials and methods}

\section{Subject selection}

The study group included 28 subjects with lumbar degenerative disease (14 subjects with L3/4 and 14 with L4/5 affected), including spondylosis, degenerative scoliosis and degenerative spondylolisthesis, in whom LLIF was performed to relieve the neuropathy (e.g. pain, gait disturbance) and low back pain at our institution from June 2014 to July 2016 (Table 1). Patients with correction surgery for adult spinal deformity with low back pain only were excluded. LLIF was performed to achieve correction and nerve decompression. After undergoing LLIF using extreme lumbar interbody fusion (XLIF) cage (XLIF ${ }^{\circledR}$; Nu Vasive Inc., California, USA) in the lateral position, the posterior side was stabilized with in situ percutaneous pedicle screws (PPS) in the prone position. PPS insertion was performed without any accompanying corrective procedure (e.g. posterior osteotomy, compression, distraction manipulation). This study was approved by the hospital's ethics committee.

\section{Imaging studies}

Each subject underwent $\mathrm{CT}$ imaging (CT machine: SOMATOM Definition AS $+{ }^{\circledR}$; SIEMENS Healthineers Inc., JAPAN, tube voltage: $120 \mathrm{kV}$, tube current: approximately $120 \mathrm{mAs}$, field of view: approximately $250 \times 180 \mathrm{~mm}$, image matrix: $512 \times 512$, slice increment: $5 \mathrm{~mm}$, slice thickness: $5 \mathrm{~mm}$ ) in a supine position preoperatively and 3 months after surgery. 3D vertebral models of the lumbar spine were created using 3D reconstruction software (Mimics ${ }^{\circledR}$; Materialise Inc., Leuven, Belgium). In the postoperative $3 \mathrm{D}$ vertebral models, pedicle screws and intervertebral cages were excluded from the models.

\section{D alignment analyses}

Posterior wall models were created from the vertebral models for 3D alignment analyses. Eigenvectors of each posterior wall were calculated to determine 3D orientation of the posterior wall. A Cartesian $(X-Y-Z)$ local coordinate system was set on each posterior wall in which an origin was set on a centroid of the posterior wall and orientation was determined by the eigenvectors. The $X, Y$ and $Z$ axes corresponded to mediolateral, posteroanterior and craniocaudal axes, respectively. The coronal $(X Z)$ plane, sagittal $(Y Z)$ plane and transverse $(X Y)$ plane were defined from 
these $X-Y-Z$ axes. The rotations of the superior vertebral body relative to the inferior vertebral body in the adjacent two vertebral bodies were expressed using Eulerian angles in a transverse plane-sagittal plane-coronal plane $(Z-X-Y)$ sequence. The wedge angle, lordosis angle and axial rotation angle were defined by the Eulerian angles in the coronal plane, sagittal plane and axial plane, respectively. A positive value of the lordosis angle was defined as lordosis and the negative value as kyphosis (Fig. 1a, b).

\section{Disc height measurement}

Three-dimensional disc height distribution was measured by the least distances between each point of the lower bony endplate of the superior vertebral body and the superior bony endplate of the inferior vertebral body, and the mean value of the least distances was determined as the disc height (Fig. 1c) [7].

\section{Spinopelvic parameter measurement}

The Cobb angle was measured on a standing anteroposterior radiographic image. Lumbar lordosis (LL), thoracic kyphosis (TK), sagittal vertical axis (SVA), sacral slope (SS), pelvic tilt (PT) and pelvic incidence (PI) were measured on standing lateral radiographic images.

\section{Statistical analyses}

The rotational angles, disc heights and spinopelvic parameters measured preoperatively and postoperatively were compared by paired $t$ tests. Correction rates of these values due to surgery were calculated by lpreoperative value - postoperative valuel/preoperative value. For the correlation between the two groups, Pearson's correlation coefficient and a $t$ test were used. Statistical significance was defined as $P<0.05$. The results were shown as mean \pm standard deviation (SD).

\section{Results}

\section{Correlations between the rotational angles (before LLIF)}

A strong positive correlation was found between the wedge angle and the axial rotation angle $(r=0.718, P<0.001)$ in the patients with lumbar degenerative disease preoperatively (Fig. 2).

\section{Effect of LLIF procedure on correction of 3D segmental deformities}

The wedge angle decreased after surgery $(P<0.001)$ from the preoperative value of $8.4^{\circ} \pm 5.4^{\circ}$ (range $0.8^{\circ}-25.6^{\circ}$ ) to the postoperative value of $3.8^{\circ} \pm 3.1^{\circ}$ (range $0^{\circ}-9.7^{\circ}$ ) by a correction rate of $55 \%\left(-4.6^{\circ} \pm 3.5^{\circ}\right)$. The lordosis angle increased after surgery $(P<0.01)$ from the preoperative value of $5.7^{\circ} \pm 5.3^{\circ}$ (range $-7.3^{\circ}$ to $17.5^{\circ}$ ) to the postoperative value of $7.8^{\circ} \pm 5.2^{\circ}$ (range $-1.1^{\circ}$ to $17.2^{\circ}$ ) by a correction rate of $37 \%\left(+2.1^{\circ} \pm 4.0^{\circ}\right)$. The axial rotation angle decreased after surgery $(P<0.001)$ from the preoperative value of $5.9^{\circ} \pm 4.2^{\circ}$ (range $0.7^{\circ}-21.1^{\circ}$ ) to the postoperative value of $3.8^{\circ} \pm 3.4^{\circ}$ (range $0.1^{\circ}-13.7^{\circ}$ ) by a correction rate of $35 \%\left(-2.1^{\circ} \pm 5.0^{\circ}\right)$ (Fig. 3$)$.
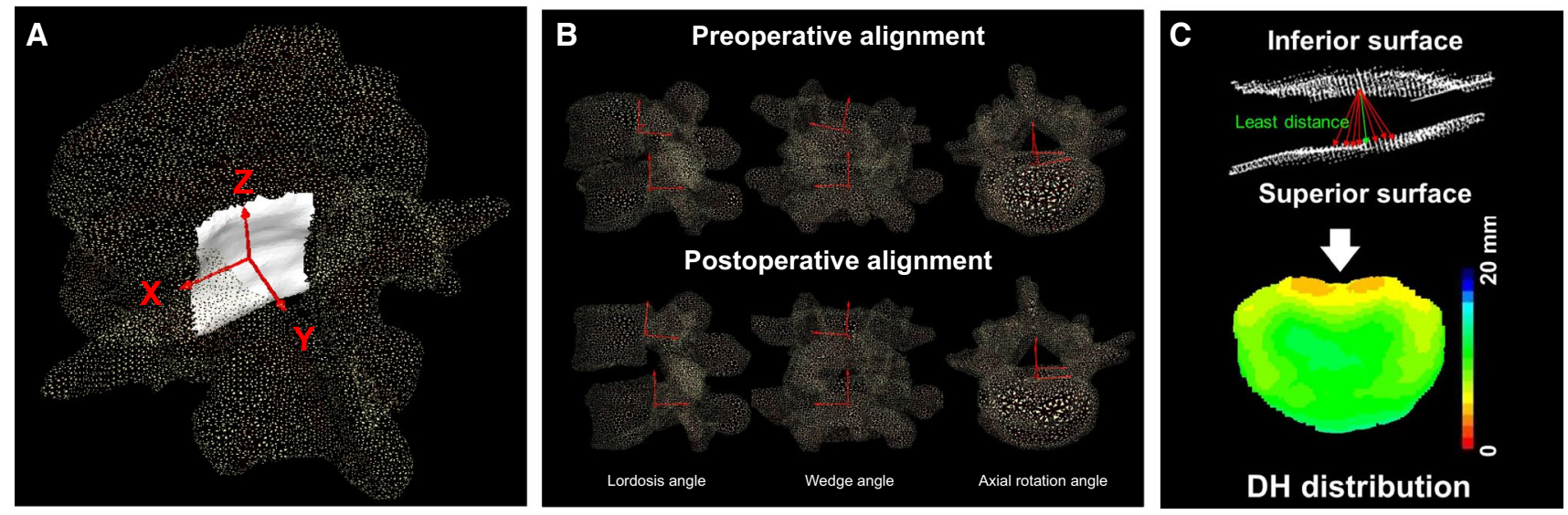

Fig. 1 a Eigenvectors of each posterior wall were calculated to determine 3D orientation of the posterior wall. A Cartesian $(X-Y-Z)$ local coordinate system was set on each posterior wall in which an origin was set on a centroid of the posterior wall and orientation was determined by the eigenvectors. b The rotations of the superior vertebral body relative to the inferior vertebral body in the adjacent two vertebral bodies were expressed using Eulerian angles. The wedge angle, lordosis angle and axial rotation angle were defined by them in the coronal plane, sagittal plane and axial plane, respectively. c Threedimensional disc height distribution was measured by the least distances between each point of the lower bony endplate of the superior vertebral body and the superior bony endplate of the inferior vertebral body, and the mean value of the least distances was determined as the disc height 


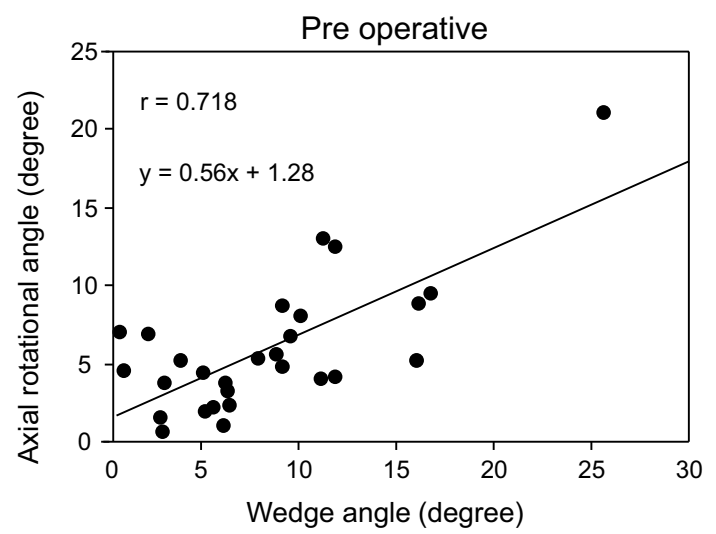

Fig. 2 A strong positive correlation was found between the wedge angle and the axial rotation angle $(r=0.718, P<0.001)$ in the patients with lumbar degenerative disease preoperatively

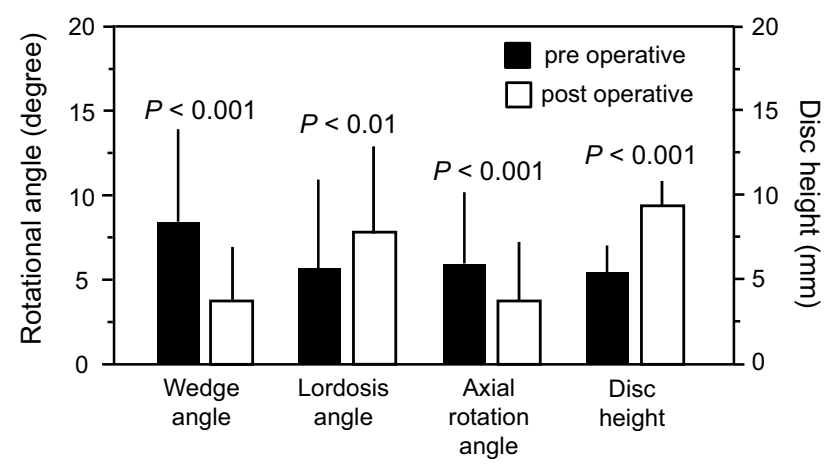

Fig. 3 Disc height and the lordosis angle increased, while the wedge and axial rotation angles decreased after LLIF

The disc height increased after surgery $(P<0.001)$ from the preoperative value of $5.4 \pm 1.5 \mathrm{~mm}$ (range $2.7-8.1 \mathrm{~mm}$ ) to the postoperative value of $9.3 \pm 1.4 \mathrm{~mm}$ (range $7.3-12.8 \mathrm{~mm})$ by a correction rate of $72 \%(3.9 \pm 1.6 \mathrm{~mm})$ (Fig. 3).

A positive correlation was found between the wedge angle and the axial rotation angle $(r=0.46, P<0.001$, Fig. 4).

\section{Effect of LLIF procedure on correction of global deformities}

The Cobb angle decreased after surgery $(P=0.035)$ from the preoperative value of $15.2^{\circ} \pm 9.7^{\circ}$ (range $2^{\circ}-40^{\circ}$ ) to the postoperative value of $11.6^{\circ} \pm 9.1^{\circ}\left(\right.$ range $\left.0^{\circ}-37^{\circ}\right)$. The LL increased after surgery $(P=0.041)$ from the preoperative value of $27.7^{\circ} \pm 18.8^{\circ}$ (range $-7^{\circ}$ to $60^{\circ}$ ) to the postoperative value of $34.4^{\circ} \pm 17.5^{\circ}$ (range $-6^{\circ}$ to $68^{\circ}$ ). The SVA decreased after surgery $(P<0.01)$ from the preoperative

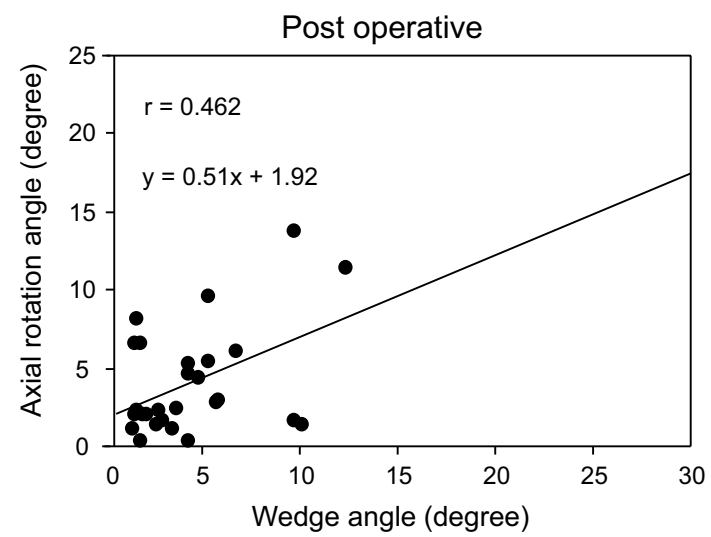

Fig. 4 A positive correlation was found between the wedge angle and the axial rotation angle $(r=0.46, P<0.001)$ in the patients with lumbar degenerative disease postoperatively

Table 2 Average values of spinopelvic parameters before and after surgery in this study

\begin{tabular}{lccc}
\hline & Preoperative & Postoperative & $P$ value \\
\hline Cobb angle & $15.2^{\circ} \pm 9.7^{\circ}$ & $11.6^{\circ} \pm 9.1^{\circ}$ & 0.035 \\
LL & $27.7^{\circ} \pm 18.8^{\circ}$ & $34.4^{\circ} \pm 17.5^{\circ}$ & 0.041 \\
TK & $25.0^{\circ} \pm 11.9^{\circ}$ & $24.8^{\circ} \pm 12.8^{\circ}$ & 0.452 \\
SS & $24.8^{\circ} \pm 10.4^{\circ}$ & $26.8^{\circ} \pm 7.6^{\circ}$ & 0.169 \\
PT & $25.6^{\circ} \pm 8.2^{\circ}$ & $23.2^{\circ} \pm 7.3^{\circ}$ & 0.074 \\
PI & $50.3^{\circ} \pm 8.2^{\circ}$ & $50.0^{\circ} \pm 8.3^{\circ}$ & 0.413 \\
SVA & $72.6 \pm 57.8 \mathrm{~mm}$ & $42.1 \pm 37.8 \mathrm{~mm}$ & $<0.01$ \\
PI-LL & $22.6^{\circ} \pm 16.3^{\circ}$ & $15.5^{\circ} \pm 15.3^{\circ}$ & 0.017 \\
\hline
\end{tabular}

value of $72.6 \pm 57.8 \mathrm{~mm}$ (range -7.5 to $40 \mathrm{~mm}$ ) to the postoperative value of $42.1 \pm 37.8 \mathrm{~mm}$ (range -15.9 to $166.1 \mathrm{~mm})$. The PI-LL decreased after surgery $(P=0.017)$ from the preoperative value of $22.6^{\circ} \pm 16.3^{\circ}$ (range $-8^{\circ}$ to $62^{\circ}$ ) to the postoperative value of $15.5^{\circ} \pm 15.3^{\circ}$ (range $-13^{\circ}$ to $52^{\circ}$ ) (Table 2).

\section{Representative case}

The patient complained of right lower extremity pain before surgery. We diagnosed L4/5 right foraminal stenosis and performed LLIF. The wedge angle decreased after surgery from the preoperative value of $11.3^{\circ}$ to the postoperative value of $4.8^{\circ}$ (correction rate $58 \%$ ). The lordosis angle increased after surgery from the preoperative value of $1.3^{\circ}$ to the postoperative value of $5.9^{\circ}$ (correction rate $354 \%$ ). The axial rotation angle decreased after surgery from the preoperative value of $13^{\circ}$ to the postoperative value of $9.5^{\circ}$ (correction rate $27 \%$ ). The disc height increased after surgery from the preoperative value of $4.3 \mathrm{~mm}$ to the postoperative value of $10.5 \mathrm{~mm}$ 


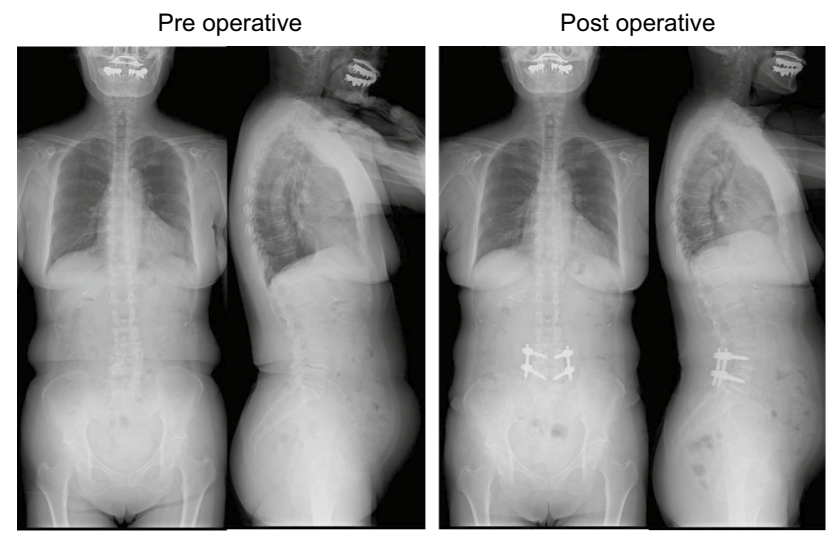

Fig. 5 Standing anteroposterior and lateral radiographic images before and after surgery in the representative case. The disc height and the lordosis angle increased, while the wedge and axial rotation angles decreased after LLIF. The Cobb angle and PI-LL decreased, while the LL increased

(correction rate 144\%) (Fig. 5). Postoperatively, the right leg pain was alleviated.

\section{Discussion}

The present study demonstrated that the strong correlations between the wedge angle and the axial rotation angle existed in the patients with lumbar degenerative disease and the LLIF procedure restored the disc height and corrected 3D deformities in three anatomical planes using the patient-specific 3D CT models created by preoperative and postoperative CT scanning. The 3D analyses of the lumbar alignment allowed accurate quantitative measurements of 3D rotational deformities in the patients with lumbar degenerative disease and the correction of these rotational deformities by the LLIF which is difficult to measure in the two-dimensional plane radiograms.

Longitudinal studies to look at progression of the spinal deformity by evaluating Cobb angle and rotational deformity by such as Nash and Moe's method which determined the grade ( 0 to IV) reported that Cobb angle increased by more than $10^{\circ}$ in grade II and III cases in patients with adult spinal deformity who had follow-up for at least 10 years [3]. These studies indicated that the axial rotation is one of the relevant factors of progression of the DLS patients [8,9]. Korovessis et al. and Ferrero et al. stated that intervertebral disc space asymmetry that occurs, followed by rotatory subluxation, including intervertebral lateral slip and rotation, causes de novo degenerative scoliosis as the 3D deformity mechanism of the spine $[10,11]$. It is possible that the deformity of the vertebral bodies, the form of the facet joints, the angle of the cage, the position of cage after insertion, the shape of the adjacent intervertebral space, osteoporosis, among other factors, might be related $[12,13]$. The quantitative
3D analysis on the segmental axial rotation using patientspecific 3D models shown in the present study would provide more accurate prediction of future progression of the spinal deformity in DLS. Future longitudinal studies will be warranted to demonstrate the benefit of this technology.

Surgical correction of the combined wedge and rotational deformity is important for improving clinical symptoms derived from intervertebral foramina and spinal canal stenosis. Wiktor et al. reported that they obtained $29.3 \%$ rotational correction of DLS by direct vertebral rotation method using pedicle screws and corrective connection devices [14], but we could find no reports of rotation correction with LLIF. In the present study, we achieved rotational correction of $35 \%$ with the LLIF procedure. It should be noted that intentional correction of the axial rotation from the posterior side or dissociated facet joints was not performed in the present study.

In this study, not only was the local lordosis angle increased by LLIF but the global deformity was affected. The LL increased, and the SVA and PI-LL decreased, with the increase in LL large relative to the increase in the local lordosis angle. These improvements in sagittal parameters are unlikely to be the result of local anatomical changes alone. We surmised that they came close to the patient's original posture because the back and leg pain was relieved. The Cobb angle in the coronal plane was also corrected, correlated with the parallel reduction in a single intervertebral disc space. These alterations in the global alignment would produce better clinical results along with the local effects of the single-level correction.

The present study showed the positive correlation between the wedge correction angle and the axial rotation correction angle. Interestingly, this correlation is similar to the correlation between wedge deformation angle and axial rotation deformation angle shown preoperatively with the similar correlation equation. Because only distraction force was applied to restore the disc height and no external torque was applied to correct the axial rotation in the present study, wedge and axial rotational movements during progression of deformity or correction procedure can be described as a "coupled motion".

The coupled motion in lumbar lateral bending has been observed in several studies [15-17]. In lateral bending, the inferior articular process glides superior direction in reference to the superior articular process of the inferior vertebra on the convex side of the spinal curve and opposite direction on the concave side [18]. Schendel et al. reported that lateral bending motion was coupled with axial rotation (i.e. left lateral bending was associated with axial rotation which loads the right facet) and the facet resultant contact force location in left lateral bending was in the same area as that for right axial torsion [15]. The authors suggested that the axial rotation component associated with lateral bending could be partially responsible for facet loading [15]. During 
correction of the wedge deformity, spinal ligamentotaxis by anterior and posterior longitudinal ligaments may also contribute to the coupled motion $[19,20]$.

There are some limitations in our study. We did not evaluate the installation position of the XLIF cage. The position of the cage could affect not only the lordosis angle but also the wedge angle and axial rotation angle. The CT scans used for postoperative evaluation in our study were obtained after both the cage and PPS had been inserted. Therefore, the contribution of correction achieved by in situ PPS fixation cannot be ignored. Because we did not consider facet degeneration, disc degeneration, segmental stiffness or size or distribution of osteophytes, it is unclear whether the correction effect will be reproducible when this procedure is performed on other patients.

Future studies on 3D geometry and kinematics of the facet joint will be needed to understand the wedge and axial rotational coupled deformation in the patients with lumbar degenerative disease and the coupling effects in correction of the 3D deformities. Evaluation of deformity and/or dislocation of the facet joint may also be needed to predict the reduction effects of the LLIF in the future studies.

\section{Conclusion}

The present study demonstrated positive correlations between the wedge deformity and the axial rotational deformity in 28 patients with lumbar degenerative disease who underwent LLIF surgery using patient-specific 3D CT models. The axial rotational deformity was simultaneously corrected with LLIF only by leveling the intervertebral wedge deformity via cage insertion. The coupled motion caused by the facet joint and ligamentotaxis may contribute to achieve intervertebral correction of both wedge and axial rotational deformities only by inserting a cage during LLIF; however, future studies will be required to understand mechanisms of the coupling effects in correction of the 3D deformities by LLIF.

\section{Compliance with ethical standards}

Conflict of interest The authors declare that they have no conflict of interest.

Open Access This article is licensed under a Creative Commons Attribution 4.0 International License, which permits use, sharing, adaptation, distribution and reproduction in any medium or format, as long as you give appropriate credit to the original author(s) and the source, provide a link to the Creative Commons licence, and indicate if changes were made. The images or other third party material in this article are included in the article's Creative Commons licence, unless indicated otherwise in a credit line to the material. If material is not included in the article's Creative Commons licence and your intended use is not permitted by statutory regulation or exceeds the permitted use, you will need to obtain permission directly from the copyright holder. To view a copy of this licence, visit http://creativecommons.org/licenses/by/4.0/.

\section{References}

1. Faraj SSA, Holewijn RM, Miranda L et al (2016) De novo degenerative lumbar scoliosis: a systematic review of prognostic factors for curve progression. Eur Spine J 25:2347-2358

2. Faraj SSA, Haanstra TM, Martijn H et al (2017) Functional outcome of non-surgical and surgical management for de novo degenerative lumbar scoliosis: a mean follow-up of 10 years. Scoliosis Spinal Disord 5(12):35

3. Nash CL Jr, Moe JH (1969) A study of vertebral rotation. J Bone Jt Surg Am 51:223-229

4. Oliveira L, Marchi L, Coutinho E et al (2010) A radiographic assessment of the ability of the extreme lateral interbody fusion procedure to indirectly decompress the neural elements. Spine (Phila Pa 1976) 35(26 Suppl):S331-S337

5. Elowitz EH, Yanni DS, Chwajol M et al (2011) Evaluation of indirect decompression of the lumbar spinal canal following minimally invasive lateral transpsoas interbody fusion: radiographic and outcome analysis. Minim Invasive Neurosurg 54(5-6):201-206

6. Dahdaleh NS, Smith ZA, Snyder LA et al (2014) Lateral transpsoas lumbar interbody fusion: outcomes and deformity correction. Neurosurg Clin N Am 25(2):353-360

7. Watanabe S, Inoue N, Yamaguchi T et al (2012) Three-dimensional kinematic analysis of the cervical spine after anterior cervical decompression and fusion at an adjacent level. Eur Spine J 21:946

8. Pritchett JW, Bortel DT (1993) Degenerative symptomatic lumbar scoliosis. Spine (Phila Pa 1976) 18(6):700-703

9. Sapkas G, Efstathiou P, Badekas AT et al (1996) Radiological parameters associated with the evolution of degenerative scoliosis. Bull Hosp Jt Dis 55:40-45

10. Korovessis P, Piperos G, Sidiropoulos P et al (1994) Adult idiopathic lumbar scoliosis. A formula for prediction of progression and review of the literature. Spine 19:1926-1932

11. Ferrero E, Lafage R, Challier V (2015) Clinical and stereoradiographic analysis of adult spinal deformity with and without rotatory subluxation. Orthop Traumatol Surg Res 101:613-618

12. Kuner EH, Kuner A, Schlickewei W, Mullaji AB (1994) Ligamentotaxis with an internal spinal fixator for thoracolumbar fractures. J Bone Jt Surg Br 76(1):107-112

13. Anand N, Cohen RB, Cohen J et al (2017) The influence of lordotic cages on creating sagittal balance in the CMIS treatment of adult spinal deformity. Int J Spine Surg 30(11):23

14. Wiktor U, Michal JW, Wojciech J et al (2017) The impact of direct vertebral rotation (DVR) on radiographic outcome in surgical correction of idiopathic scoliosis. Arch Orthop Trauma Surg 137:879-885

15. Schendel MJ, Wood KB, Buttermann GR et al (1993) Experimental measurement of ligament force, facet force, and segment motion in the human lumbar spine. J Biomech 26:427-438

16. Gregersen GG, Lucas DB (1967) An in vivo study of the axial rotation of the human thoracolumbar spine. J Bone Jt Surg Am 49:247-262

17. White AA, Panjabi MM (1978) Clinical biomechanics of the spine. J. B. Lippencott, Philadelphia

18. Jegapragasan M, Cook DJ, Gladowski DA et al (2011) Characterization of articulation of the lumbar facets in the human cadaveric spine using a facet-based coordinate system. Spine J 11:340-346 
19. Hiroyuki Y (2017) Indirect decompression in spinal surgery. J Clin Neurosci 44:63-68

20. Fujibayashi S, Hynes RA, Otsuki B et al (2015) Effect of indirect neural decompression through oblique lateral interbody fusion for degenerative lumbar disease. Spine 40(3):E175-E182
Publisher's Note Springer Nature remains neutral with regard to jurisdictional claims in published maps and institutional affiliations.

\section{Affiliations}

\section{Hiroto Yamaguchi ${ }^{1,2} \cdot$ Hidetoshi Nojiri $^{1}$ (D ) Kei Miyagawa ${ }^{1} \cdot$ Nozomu Inoue $^{3} \cdot$ Kazuo Kaneko $^{1}$}

1 Department of Orthopedic Surgery, Juntendo University School of Medicine, 3-1-3 Hongo, Bunkyo-ku,

Tokyo 113-8421, Japan

2 Department of Orthopedic Surgery, Juntendo Tokyo Koto Geriatric Medical Center, Tokyo, Japan

\author{
Department of Orthopedic Surgery, Rush University, \\ Chicago, IL, USA
}

